\title{
Activation of Prefrontal Cortex by Transcranial Direct Current Stimulation Reduces Appetite for Risk during Ambiguous Decision Making
}

\author{
Shirley Fecteau, ${ }^{1}$ Alvaro Pascual-Leone, ${ }^{1}$ David H. Zald, ${ }^{2}$ Paola Liguori, ${ }^{3}$ Hugo Théoret, ${ }^{4}$ Paulo S. Boggio, ${ }^{3}$ and \\ Felipe Fregni ${ }^{1}$ \\ ${ }^{1}$ Berenson-Allen Center for Noninvasive Brain Stimulation, Department of Neurology, Beth Israel Deaconess Medical Center and Harvard Medical School, \\ Boston, Massachusetts 02215, ${ }^{2}$ Department of Psychology, Vanderbilt University, Nashville, Tennessee 37203, ${ }^{3}$ Mackenzie University, 05508 São Paulo, \\ Brazil, and ${ }^{4}$ Université de Montréal and Hôpital Sainte-Justine, Montréal, Québec, Canada H3T 1C5
}

As adult humans, we are continuously faced with decisions in which proper weighing of the risk involved is critical. Excessively risky or overly cautious decision making can both have disastrous real-world consequences. Weighing of risks and benefits toward decision making involves a complex neural network that includes the dorsolateral prefrontal cortex (DLPFC), but its role remains unclear. Repetitive transcranial magnetic stimulation studies have shown that disruption of the DLPFC increases risk-taking behavior. Transcranial direct current stimulation (tDCS) allows upregulation of activity in the DLPFC, and we predicted that it might promote more cautious decision making. Healthy participants received one of the following treatments while they performed the Balloon Analog Risk Task: (1) right anodal/left cathodal DLPFC tDCS, (2) left anodal/ right cathodal DLPFC tDCS, or (3) sham tDCS. This experiment revealed that participants receiving either one of the bilateral DLPFC tDCS strategies adopted a risk-averse response style. In a control experiment, we tested whether unilateral DLPFC stimulation (anodal tDCS over the right or left DLPFC with the cathodal electrode over the contralateral supraorbital area) was sufficient to decrease risk-taking behaviors. This experiment showed no difference in decision-making behaviors between the groups of unilateral DLPFC stimulation and sham stimulation. These findings extend the notion that DLPFC activity is critical for adaptive decision making, possibly by suppressing riskier responses. Anodal tDCS over DLPFC by itself did not significantly change risk-taking behaviors; however, when the contralateral DLPFC was modulated with cathodal tCDS, an important decrease in risk taking was observed. Also, the induced cautious decision-making behavior was observed only when activity of both DLPFCs was modulated. The ability to modify risk-taking behavior may be translated into therapeutic interventions for disorders such as drug abuse, overeating, or pathological gambling.

Key words: transcranial direct current stimulation; prefrontal cortex; balloon analog risk task; humans; decision making; risk

\section{Introduction}

Decisions require careful weighing of the risks and benefits. Poor decision making, either because of an excessively risky or an overly cautious approach, can have deleterious consequences for health, safety, and financial well being. Adaptive decision making requires, among others, the assessment and selection of risky and less-risky behavioral choices and the prediction and evaluation of the potential outcomes (Ernst and Paulus, 2005) to reach a choice consistent with the individual's personality, goals, and desires.

Received Sept. 14, 2006; revised March 2, 2007; accepted March 9, 2007

This work was supported by a fellowship from the Fonds de Recherche en Santé du Québec to S.F., National Institutes of Health (NIH) Grants (K24 RR018875, R01-MH069898, R01-DC05672, R01-NS 47754, R01-NS 20068, R01-EY12091, R01-EB 005047, R03 DK071851), and the Harvard-Thorndike General Clinical Research Center (NCRR M01 RR01032). F.F. was supported by NIH Grant DK071851-01 and the Harvard University David Rockefeller CenterJorge Paulo Lemann Fellowship.

Correspondence should be addressed to Dr. Felipe Fregni or Dr. Alvaro Pascual-Leone, Center for Noninvasive Brain Stimulation, Department of Neurology, Beth Israel Deaconess Medical Center, 330 Brookline Avenue KS 452, Boston, MA 02215. E-mail: ffregni@bidmc.harvard.edu or apleone@bidmc.harvard.edu.

D0I:10.1523/JNEUROSCI.0314-07.2007

Copyright $\odot 2007$ Society for Neuroscience $\quad$ 0270-6474/07/276212-07\$15.00/0
The study of patients with focal brain lesion has greatly contributed to the understanding of the neural basis of decision making (Bechara et al., 1994; Damasio et al., 1996) and has lead to the elaboration of theoretical models (Damasio et al., 1996) and well designed neuroimaging experiments (Rogers et al., 1999; Paulus et al., 2001; Sanfey et al., 2003). Clinical and neuroimaging studies reveal the involvement of a distributed bihemispheric, corticosubcortical network in decision making (Ernst et al., 2002; Ernst and Paulus, 2005; Fishbein et al., 2005; Krain et al., 2006; Navqi et al., 2006). The dorsolateral prefrontal cortex (DLPFC) is part of this network (Manes et al., 2002; Clark et al., 2003) and appears particularly involved in decision making when choices are ambiguous (Krain et al., 2006).

Neuroimaging studies are useful in establishing correlations between brain activations and risk-taking processes, but they do not provide information regarding whether a given region is necessary to the resulting behavior. Noninvasive brain stimulation techniques, such as repetitive transcranial magnetic stimulation (rTMS), allow the study of the behavioral consequences of an externally induced brain activation or inactivation in healthy 
subjects, thus establishing a causal relationship between brain and behavior without most of the confounds inherent to natural lesion studies (Rafal, 2001; Robertson et al., 2003).

van't Wout et al. (2005) and Knoch et al. (2006) have used low-frequency rTMS to transiently suppress activity in the DLPFC and provide evidence for its causal role in risk-taking behavior. Transcranial direct current stimulation (tDCS) has some advantages as compared with rTMS because it induces a stronger modulatory effect on brain activity (Nitsche and Paulus, 2001; Romero et al., 2002), provides better conditions to online testing, and allows for a reliable sham condition (Gandiga et al., 2006). In addition, anodal tDCS increases excitability in the targeted brain region (Wassermann and Grafman, 2005). These excitability shifts during stimulation are believed to be resulting from subthreshold neuronal membrane depolarization (Nitsche and Paulus, 2000; Liebetanz et al., 2002; Priori, 2003). The most probable mechanism is that resting membrane modulation is caused by the electrical current flow, which involves opening or closing of voltage-gated ion channels (Purpura and McMurtry 1965; Nitsche et al., 2003a). This externally induced enhancement of excitability can transiently modulate behavioral performance in healthy humans (Nitsche et al., 2003b; Antal et al., 2004; Kincses et al., 2004; Fregni et al., 2005; Marshall et al., 2005).

It remains unknown whether upregulating activity using noninvasive brain stimulation could result in more cautious decision making. This is an important issue because a high propensity to risk-taking decision making appears linked to an increased vulnerability for addictive pathological behavior (Epstein et al., 2006), and noninvasive neuromodulation may thus have potential therapeutic uses in various addiction disorders. Indeed, modulation of cortical excitability in frontal areas can suppress craving for nicotine (Eichhammer et al., 2003), food (Uher et al., 2005), and cocaine (Camprodon et al., 2007).

The goal of the present study was to modulate decision making by upregulating cortical excitability over the DLPFC in healthy adults. We used the Balloon Analog Risk Task (BART; Lejuez et al., 2002) as a measure of risk-taking behavior. Based on previous findings showing that inhibition of DLPFC activity results in increased risk taking (Knoch et al., 2006), and enhanced DLPFC activity appears to be associated with decision-making behaviors (for review, see Ernst and Paulus, 2005; Krain et al., 2006), we hypothesized that modulating DLPFC activity with anodal stimulation would lead to diminished risk-taking behavior. We therefore initially performed an experiment investigating whether bilateral stimulation of the DLPFC (anodal stimulation of the right and cathodal stimulation of the left DLPFC or vice versa) would change risk-taking behavior as compared with sham stimulation (study 1). To explore whether these effects were caused mainly by DLPFC anodal stimulation or, alternatively, whether DLPFC cathodal stimulation was also contributing to the observed effect, we performed a subsequent experiment in which we tested whether unilateral DLPFC stimulation by placing the anodal electrode over either the right or the left DLPFC with the cathodal electrode over the contralateral supraorbital region (rather than the contralateral DLPFC) resulted in a different risk-taking behavior compared with sham stimulation (study 2). Finally, we included the color-word Stroop task as a control experiment to test the possible effect of tDCS over the DLPFC on the neighboring regions, such as the orbitofrontal area, which seems to be involved in inhibitory control functions (Elliot and Deakin, 2005). A tDCS-induced effect on inhibitory functions would lead to difference in performance on the BART that would not be related to risk-taking behaviors per se; therefore, participants had to perform the Stroop task before and after stimulation.

\section{Materials and Methods}

Participants. Thirty-five healthy college students (nine men; mean age, $21.0 \pm 2.8$ years; two left-handed) participated in study 1 , and 12 healthy college students (one man; mean age, $21.7 \pm 2.7$ years; one left-handed) participated in study 2. Participants were exempt from neurological or psychiatric conditions and were on no chronic medications. None of the participants had contraindications to brain stimulation. All were naive to tDCS, the BART task, and the nature of the experiment and were not explicitly informed of the experimental variable tested. Participants gave informed written consent before entering the study, which was approved by the Mackenzie University ethics committee.

$B A R T$. The BART is a behavioral measure of risk taking that has convergent validity with real-world risk-related situations. Specifically, performance on the BART has been correlated with occurrence of real-world risk behaviors (Lejuez et al., 2002, 2003a,b), such as substance use (Lejuez et al., 2005), risky sexual behaviors (Lejuez et al., 2004), and delinquent behaviors (Lejuez et al., 2002, 2003a,b; Aklin et al., 2005), as well as selfreport measures of risk-related constructs such as sensation seeking, impulsivity, and deficiencies in behavioral constraints (Lejuez et al., 2002).

In the BART, participants have to make a choice in a context of increasing risk. Participants are invited to inflate a computerized balloon by pushing a "pump." The balloon can explode at any moment. Participants have to decide after each pump whether to keep pumping and risk explosion of the balloon or to stop. Participants accumulate money in a temporary bank with each pump (five cents for each pump). When the participant decides to stop pumping, the accumulated money is transferred to a permanent bank. However, if the balloon explodes, all of the money accumulated in the temporary bank is lost. Therefore, the probability of losing the money, as well as the potential loss (i.e., the amount of money), increases with each pump. Each balloon has a different explosion point. There are a total of 30 trials (balloons). For more details on the BART, please refer to Lejuez et al. (2002).

$t D C S$. Direct current was induced by two saline-soaked surface sponge electrodes $\left(35 \mathrm{~cm}^{2}\right)$ and delivered by a battery-driven, constant-current stimulator. The device used, developed by our group, is particularly reliable for double-blind studies: a switch can be activated to interrupt the electrical current while maintaining the "ON" display and showing the stimulation parameters throughout the procedure to the experimenter and participant. For technical details, contact P.S. Boggio at sboggio@colband.com.br.

In study 1, participants were randomly assigned to receive either active stimulation with the anodal electrode over the right DLPFC and the cathodal electrode over the left DLPFC (referred to as "bilateral DLPFC anodal right/cathodal left" group; $n=10$; one man), active stimulation with the anodal electrode over the left DLPFC and the cathodal electrode over the right DLPFC (referred to as "bilateral DLPFC anodal left/ cathodal right" group; $n=10$; five men), sham stimulation ( $n=10$; three men), or no stimulation (baseline condition; $n=5$; one man). Because we hypothesized a priori that performance in the BART during sham stimulation would be no different than in the "no stimulation" condition, we tested only five participants in this latter condition. For stimulation over the left DLPFC, the anodal electrode was placed over the left F3 (international EEG 10/20 system) and the cathodal electrode over the right F4. For stimulation of the right DLPFC, the polarity was reversed: the anode was placed over the F4 and the cathode over the F3. For the sham stimulation, the electrodes were placed at the same positions as for active stimulation (F3 and F4), but the stimulator was turned on only for $30 \mathrm{~s}$. Thus, participants felt the initial itching sensation associated with tDCS, but received no active current for the rest of the stimulation period. This method of sham stimulation has been shown to be reliable (Gandiga et al., 2006).

In study 2, participants were randomly assigned to receive active stimulation either with the anodal electrode over the right DLPFC and the cathodal electrode over the left supraorbital area (referred to as the "unilateral DLPFC anodal right" group; $n=6$; zero men), or with the anodal electrode over the left DLPFC and the cathodal electrode over the right supraorbital area (referred to as "unilateral DLPFC anodal left" group; $n=6$; one man). This electrode arrangement (anodal electrode over one 


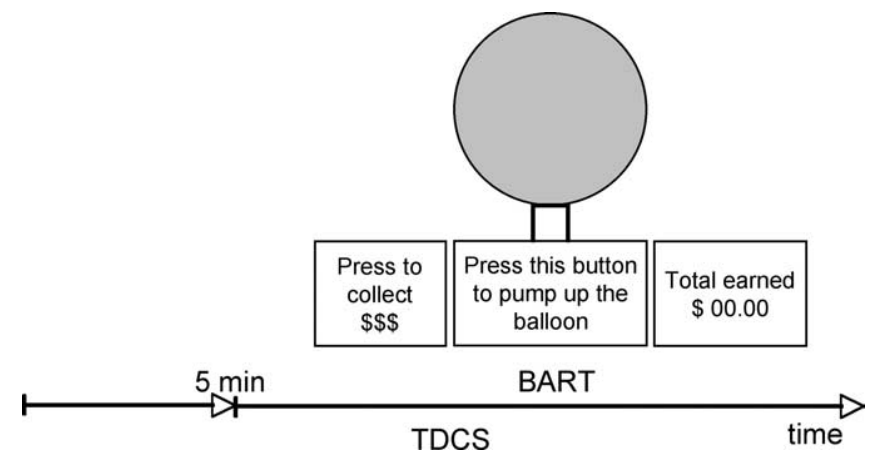

Figure 1. Schematic representation of the experimental design. Each participant started to perform the BART after receiving 5 min of stimulation. Stimulation continued throughout the BART. Before and after stimulation, they performed the Stroop task.

DLPFC with the cathodal electrode over the contralateral supraorbital area) is thought to induce unilateral modulation of one DLPFC and has been shown effective in various studies (Kincses et al., 2004; Fregni et al., 2005). For anodal stimulation over the left DLPFC, the anodal electrode was placed over the left F3 and the cathodal electrode over the right supraorbital area (international EEG 10/20 system). For anodal stimulation over the right DLPFC, the anodal electrode was placed over the right F4 and the cathodal electrode over the left supraorbital area.

For active stimulation, in both study 1 and study 2, participants received a constant current of $2 \mathrm{~mA}$ intensity. Previous studies have shown that this intensity of stimulation is safe and can be more effective than 1 mA stimulation (Iyer et al., 2005; Boggio et al., 2006). tDCS started 5 min before the task began and was delivered during the whole course of the BART, which lasted $<15$ min (Fig. 1).

The BART was conducted in an experimental room including a personal computer and the tDCS equipment. Instructions for the BART task were written so that all participants received the same instructions, and participants were invited to ask any question they may have after reading. They were given no precise information about the probability of explosion or the total amount of money acquired from previous participants. Participants were told that the subject with the highest amount of money would receive a gift certificate corresponding to 35 dollars.

Stroop task. To test the possible effect of tDCS on motor impulsiveness (i.e., the ability to inhibit a prepotent response), participants performed the Stroop task before and after stimulation. We measured Stroop interference, which is characterized by a slower response in naming incongruent words (i.e., the word "red" printed in green ink) compared with color congruent words (Stroop, 1935). Based on previous findings that lowfrequency rTMS over the DLPFC results in no effect on Stroop interference (Vanderhasselt et al., 2006; Wagner et al., 2006), we expected that tDCS over the DLPFC would have no effect on Stroop interference.

Data analysis. The main outcome measure was the adjusted number of pumps (i.e., number of pumps for balloons that did not explode), which has been advocated as the preferred dependent measure for the BART because it avoids the constraints on individual differences that occur on trials with explosions (for which there is a fixed limit of potential pumps) (Lejuez et al., 2002; Aklin et al., 2005). We also calculated the total amount of money earned. Moreover, because BART was performed during stimulation, we calculated the time course of these two measures (adjusted number of pumps and total amount of money earned).

Three participants (one in the bilateral DLPFC anodal right group, one in the bilateral DLPFC anodal left group, and one in the sham group) were excluded as outliers (2 SD above or below the mean of the group for the adjusted number of pumps). Statistical analyses were performed using SAS statistical software (version 9.1; SAS Institute, Cary, NC).

In study 1 , we first compared data from the two control groups (sham stimulation and baseline). Results revealed no significant difference between the two groups in either outcome measure (ANOVA; adjusted number of pumps, $p>0.1$; the amount of money earned, $p>0.1$ ). Therefore, data from the two control groups were collapsed and then compared with those of the two groups with active stimulation. These data were submitted to a mixed linear model in which the dependent variable was the average score of each of the two outcome measures (adjusted number of pumps, total amount of money earned) using the covariate of group (bilateral DLPFC anodal right, bilateral DLPFC anodal left, sham stimulation). In addition, we performed a mixed linear model with the covariates of group, time (10 first balloons; $10 \mathrm{~s}$ balloons; 10 last balloons), and interaction between group and time of each of the two outcome measures.

To investigate whether unilateral DLPFC stimulation might be sufficient to induce changes in decision-making behaviors, we then performed a mixed linear model in which the dependent variable was the average score of each of the two outcome measures using the covariate of group (from the two studies: bilateral DLPFC anodal right, bilateral DLPFC anodal left, sham stimulation, unilateral DLPFC anodal right, and unilateral DLPFC anodal left). We also performed a mixed linear model with the covariates of group (from the two studies), time, and interaction between group and time for each of the two outcome measures (adjusted number of pumps, total amount of money earned). If appropriate, post hoc analyses were performed with correction for multiple comparisons. Results with a $p$ value $\leq 0.05$ were considered significant for all statistical analyses.

\section{Results}

None of the participants experienced adverse effects during or after tDCS. Participants perceived a slight itching sensation under the electrodes during approximately the first $30 \mathrm{~s}$ of stimulation. Subjects in the sham stimulation group were truly blinded to the nature of stimulation, reported the same initial itching sensation, and when explicitly asked, believed to have undergone real stimulation. There was no significant difference in the amount of time needed to perform the BART between the five groups of stimulation $\left(F_{(4,39)}=2.46 ; p=0.06\right)$.

\section{Study 1}

The goal of study 1 was to investigate the effects of DLPFC modulation on decision-making behaviors. Examination of the BART data (the average number of pumps on balloons that did not explode) indicates a main effect of group $\left(F_{(2,29)}=7.90 ; p=\right.$ $0.002)$. As illustrated in Figure $2 a$, participants who received bilateral DLPFC anodal right or bilateral DLPFC anodal left stimulation, pumped less number of times than participants who received sham stimulation.

We also observed a significant interaction between group and time $\left(F_{(6,58)}=3.62 ; p=0.0041\right)$. Participants who received sham stimulation showed a substantial increase in risk taking with time (they pumped more balloons toward the end as compared with the beginning of the experiment), as shown in Figure $2 b$. In contrast, participants receiving real stimulation only showed a small increase with time.

For the total amount of money earned, there was a significant group difference $\left(F_{(2,29)}=13.29 ; p=0.001\right)$, and in a subsequent model, we observed a significant interaction between group and time $\left(F_{(6,58)}=6.40 ; p<0.001\right)$. Figure 3 shows that participants receiving bilateral DLPFC anodal right or bilateral DLPFC anodal left stimulation earned less money compared with those receiving sham stimulation.

We performed an additional analysis to explore whether there was a difference between the two groups receiving active stimulation (bilateral DLPFC anodal right or bilateral DLPFC anodal left) on the main outcome measure. We found no significant difference between the two groups for the adjusted number of pumps $(p>0.6)$.

We tested a possible impact of gender on decision making. There was no significant difference between women receiving real stimulation over the left or right hemisphere $(p>0.8)$, or 

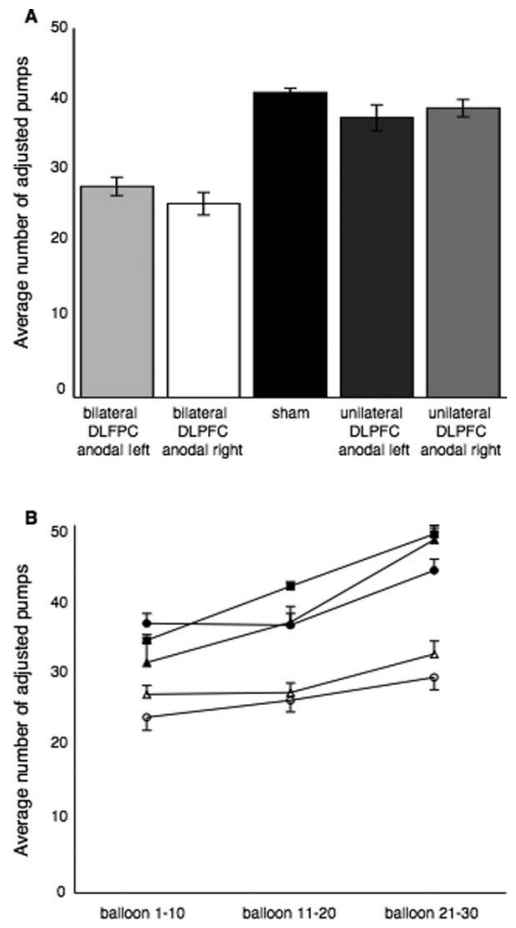

Figure 2. $\quad A, B$, Graphic display of the average number of adjusted pumps (the total pumps of the balloon that did not explode) for each group $(\boldsymbol{A})$ and the average number of adjusted pumps for each group and time period (B) (the first 10 balloons, $10 \mathrm{~s}$ balloons, and 10 last balloons). Open triangle, Bilateral DLPFC anodal left/cathodal right; open circle, bilateral DLPFC anodal right/cathodal left; filled triangle, unilateral DLPFC anodal left group; filled circle, unilateral DLPFC anodal right group; filled square, sham group. Error bars indicate SEM.
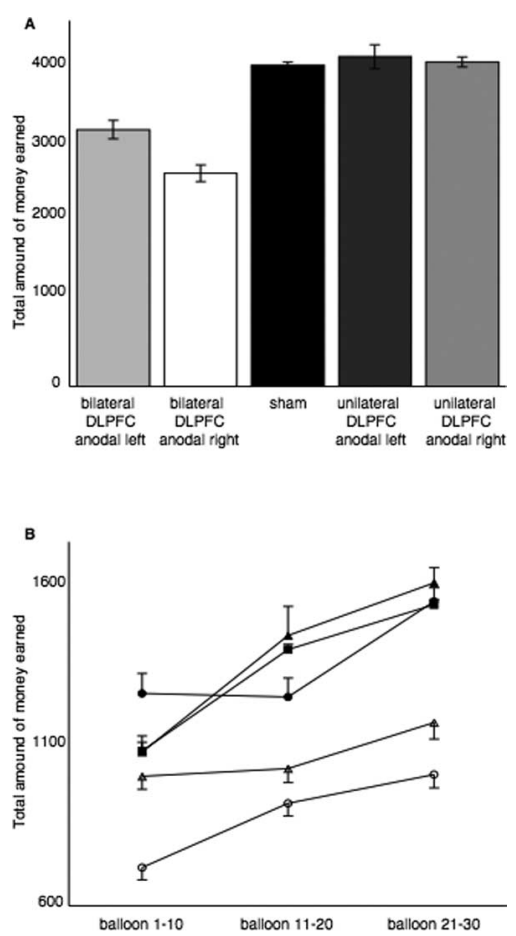

Figure 3. $A, B$, Graphic display of the total amount of money earned for each group $(\boldsymbol{A})$ and the total amount of money earned for each group and time period (B) (the first 10 balloons, $10 \mathrm{~s}$ balloons, and 10 last balloons). Open triangle, Bilateral DLPFC anodal left/cathodal right; open circle, bilateral DLPFC anodal right/cathodal left; filled triangle, unilateral DLPFC anodal left group; filled circle, unilateral DLPFC anodal right group; filled square, sham group. Error bars indicate SEM.

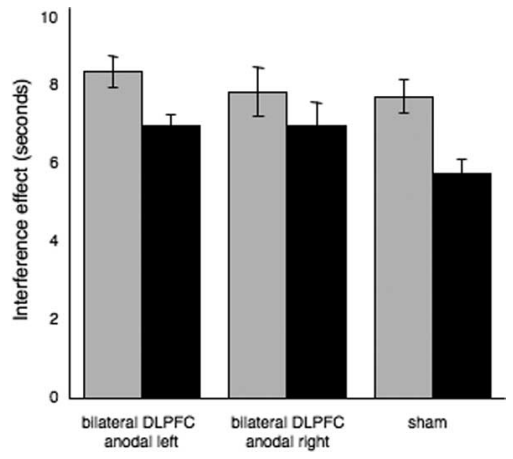

Figure 4. Stroop interference (in seconds) for three groups of stimulation before and after stimulation. Gray columns, Pre-tDCS; black columns, post-tDCS. Error bars indicate SEM.

between men and women receiving real stimulation over the right DLPFC $(p>0.9)$ for the adjusted number of pumps. Comparison between men and women receiving stimulation over the left hemisphere was not possible because of the small sample size.

The results of the Stroop task (our control condition) showed a trend effect of time of assessment on performance (repeatedmeasures ANOVA; $p=0.064$ ), reflecting faster color naming on the second assessment in all groups, likely because of repeated testing (Fig. 4). No significant group difference $(p>0.7)$ or interaction between time and group $(p>0.8)$ were observed.

\section{Study 2}

The goal of this study was to test whether unilateral DLPFC stimulation might be sufficient to modulate decision-making behaviors. We found a main effect of group $\left(F_{(4,39)}=4.23 ; p=0.0062\right)$ for the average number of pumps on balloons that did not explode. Participants with bilateral DLPFC tDCS with an anode over the right or the left DLPFC pumped less number of times than participants who received unilateral DLPFC tDCS with an anode over the right or the left DLPFC and those with sham stimulation (Fig. 2a). In addition, the average number of adjusted pumps was significantly different with regards to time $\left(F_{(2,78)}=\right.$ $13.89 ; p<0.0001)$, whereas the interaction between group and time did not reach significance $\left(F_{(8,78)}=8.83 ; p=0.3702\right)$. As shown in Figure $2 b$, all participants showed an increase in risk taking with time (they pumped the balloons more times toward the end as compared with the beginning of the experiment). For the total amount of money earned, we also found a significant group difference $\left(F_{(4,39)}=6.27 ; p=0.0005\right)$ and an effect of time $\left(F_{(2,78)}=25.64 ; p<0.0001\right)$, but no significant interaction between group and time $\left(F_{(8,78)}=1.02 ; p=0.4295\right)($ Fig. 3a,b).

\section{Discussion}

Overall, we found that participants receiving bilateral DLPFC tDCS with an anodal electrode over the right or the left DLPFC (with cathodal electrode over the homologous area of the contralateral hemisphere) displayed a conservative, risk-averse response style, making fewer pumps on the BART than those with sham stimulation and those with unilateral DLPFC stimulation (either right or left anodal electrode stimulation of the DLPFC with cathodal electrode over the contralateral supraorbital area). This difference between groups in decision making increased with time: participants receiving sham stimulation showed a progression toward making significantly more pumps as the task progressed, whereas those receiving bilateral DLPFC tDCS with anodal and cathodal tDCS over the right or the left DLPFC only 
modestly increased their number of pumps. Our findings support previous evidence showing that the DLPFC is causally involved in modulating decision making. In addition, this is the first study showing that neuromodulation can actually lead to more cautious choices in decision making in healthy subjects.

van't Wout et al. (2005) found that suppression of activity in the DLPFC using low-frequency rTMS resulted in an altered decision-making strategy. Specifically, they observed that as compared with sham stimulation, real rTMS to the right DLPFC resulted in accepting more frequently unfair offers and taking longer to refuse unfair offers. More recently, Knoch et al. (2006) reported that suppression of activity in the right but not the left DLPFC with low-frequency rTMS made participants choose high-risk prospects more often. Our results are partially in line because anodal stimulation over the right DLPFC and cathodal stimulation over the left DLPFC induced changes in the risktaking behaviors. However, cathodal stimulation over the right and anodal stimulation over the left DLPFC also induced a decrease in risk-taking behaviors. In both groups, participants with enhanced activation of the DLPFC adopt a risk-averse response style. These apparently contrary results from both studies might be because of the task and the method of stimulation involved.

In the present work, bilateral DLPFC tDCS (regardless of whether the anode was placed left or right) effectively modulated decision making. Enhancing DLPFC activity diminished risktaking behaviors but only when coupled with an inhibitory modulation over the contralateral DLPFC. Unilateral DLPFC excitatory stimulation (with the cathodal electrode over the contralateral supraorbital area) was not sufficient to modulate decision-making behaviors. One possible explanation is that the effects are mediated by the balance of activity across the hemispheres and that tDCS exerts its effect by altering the relative balance of the two DLPFCs: relative hyperactivation of one DLPFC and suppression of cortical excitability of the contralateral DLPFC. There may be a critical cross-hemisphere interplay between the right and left DLPFC during decision making that is altered by the bilateral electrode placement. In contrast, when either DLPFC is targeted alone, the effects are lost. Finally, because no effect on decision-making behaviors was observed when stimulation was restricted to one DLPFC (anodal electrode over one DLPFC with the cathodal electrode over the contralateral supraorbital area), an electrode arrangement that appears to be effective for DLPFC stimulation and for stimulating the contralateral frontopolar cortex (Kincses et al., 2004; Fregni et al., 2005), this suggests that the frontopolar cortex is not involved in decision-making behaviors as tested in the present study.

Decision-making tasks can be divided into those involving risk and those involving ambiguity (Bechara et al., 2005). Decisions involving risk reflect situations in which probabilities are known and the lower probability outcome is worth more than the higher probability outcome. In contrast, in decisions involving ambiguity, the probabilities are unknown. Some researchers, such as Krain et al. (2006), add an additional criterion for ambiguity, in which the rewards associated with the two choices are equal. However, this second criterion is not universally applied, and a condition of ambiguity can be viewed as any situation in which the likelihood of one or more of the payoffs occurring is not fully specified (Smith et al., 2002; Zak, 2004). The BART meets this broader definition of an ambiguous decision in that one of the choices (to make another pump) has unknown probabilities. At each decision point, the participant has to make a decision between a chance for an incremental gain or potentially larger losses with unknown probabilities versus a $100 \%$ certainty of no loss but no additional gain. The meta-analysis from Krain et al. (2006), which includes thirteen neuroimaging studies, suggests the preferential involvement of the orbitofrontal cortex in risky decision making, whereas the DLPFC appears to be more strongly involved in ambiguous decision making. Our results support findings from Krain et al. (2006), indicating that the DLPFC plays a role in ambiguous decision making.

Conflicting results have been reported regarding the relative contribution of the right and the left DLPFC in decision-making behaviors. It has been reported that patients with a unilateral ventromedial prefrontal cortex lesion to the right hemisphere display abnormal risk-taking behavior as compared with those with a lesion in the left hemisphere and healthy subjects (Tranel et al., 2002). However, two recent studies with a greater sample size observed that patients with lesions to the left ventromedial prefrontal cortex also display abnormal risk-taking behaviors (Clark et al., 2003; Fellows and Farah, 2005). In addition, the recent meta-analysis of Krain et al., (2006) revealed that risky and ambiguous decision making elicited activity bilaterally in the prefrontal cortex (mainly orbitofrontal and DLPFC).

In the present study, we found no differences in the left-sided and right-sided effects of tDCS on risk-taking behavior, whereas Knoch et al. (2006) found that right-sided but not left-sided rTMS had an impact on performance in the risk task. One possible explanation for this discrepancy in results is the task used. The risk task tested by Knoch et al. (2006) involves the contrast of differentially sized rewards under conditions where the probability is known, but the BART involves selection under conditions of ambiguity. It is plausible that only the right hemisphere plays a role in the risk task, whereas both hemispheres influence the ambiguity situation. An alternative explanation for the differential findings after left DLPFC stimulation between the two studies is the nature of stimulation (tDCS vs rTMS) and the direction of modulation (facilitation vs inhibition). Finally, the left-sided effect might be because of a gender difference. Some studies have reported that right-sided prefrontal lesions could yield to defects in men's but not women's social conduct, emotional processing, and decision making (Tranel et al., 2005). This may suggest that there might be laterality differences for risk-taking impact of DLPFC stimulation in men and women. Specifically, modulation of decision-making behaviors might be greater for men receiving stimulation over the right hemisphere but greater for women receiving stimulation over the left hemisphere. However, our results indicate that the effect of stimulation over the left DLPFC was not entirely driven by women participants, because there was no significant difference between women receiving stimulation over the left or the right hemisphere. Although we did not find a gender difference in the BART measures, the ratio between men and women was not 50:50. Additional studies should specifically explore whether there is a gender difference in decision making in regards to stimulation.

In general, under conditions of ambiguity, healthy humans show a risk-averse response style, often forgoing potential rewards in favor of a sure thing. This is indeed the case with the BART, where most people perform in a manner that is suboptimal because of an overly conservative risk-averse response style. Over time, performance tends to change, and with continued play, subjects become more willing to increase their risk. In our study, all participants show such a trend. However, participants with bilateral DLPFC stimulation (with anodal and cathodal electrodes over the right or left DLPFC) showed only a modest increase in the number of pumps as compared with participants with sham stimulation. In the BART, the probabilities can only be estimated through trial and error learning by playing of the game 
itself. Therefore, early decisions must be made under a high degree of uncertainty, and may therefore emphasize ambiguity tolerance and aversion, whereas in later trials, when the probabilities may be approximately known, the task may start to reflect tolerance or aversion for making lower probability choices to gain a larger reward (i.e., decisions based on risk). Participants receiving active modulation of bilateral DLPFC may be performing in such an ambiguity-averse manner that they fail to gain a good approximation of the actual probabilities of the explosion of the balloon. It is also possible that the initial risk-averse style displayed by participants receiving active modulation of bilateral DLPFC may propagate to later trials because they do not get much opportunity to get a more informed estimate of the probability of explosions. In other words, the stimulation appears to produce such a risk-averse bias that it prevents the participants from acquiring information that would lead to a more optimal response strategy.

An intriguing interpretation of these data are that participants in the sham stimulation group were not necessarily more risktaking but instead performed at a more optimal level. Indeed, participants receiving active stimulation earned significantly less money than those receiving sham stimulation. The most likely explanation for this finding is that earning less money in the BART is intrinsically related to the smaller number of times the balloons have been pumped. As shown in previous studies, the more risk taking the participants, the more money they earned (Lejuez et al., 2003a). In that sense, in healthy participants, anodal tDCS over the DLPFC coupled with cathodal stimulation over the contralateral DLPFC may actually be detrimental to certain specific behaviors (as seen here in the amount of money earned on the task), because risk taking in this case might not be, per se, maladaptive. Interestingly, smokers earn more money than nonsmokers, using the BART (Lejuez et al., 2003a). However, at the other end of the spectrum, findings from van't Wout et al. (2005) and Knoch et al. (2006) suggest that inhibiting activity in the right DLPFC results in making the wrong decision more often (i.e., accepting more frequently unfair offers) (van't Wout et al., 2005) and taking excessive risks that lead to less gain (Knoch et al., 2006). Here, participants made safer choices, resulting in earning less money.

The low spatial resolution of tDCS is an inherent limitation of this noninvasive brain stimulation technique. Stimulation of the DLPFC may coactivate other frontal regions such as the orbitofrontal/ventromedial cortex because they are densely interconnected (Ghashghaei and Barbas, 2002) and spatially close. The orbitofrontal region appears to be especially involved in inhibitory control functions (Elliot and Deakin, 2005) but the effects on risk taking reported here seem unlikely to be because of distal impact on this region. If this were the case, we would have expected a between-group difference in task completion duration if abnormal inhibition were present, which was not the case. In addition, no group difference on the Stroop interference task was observed after stimulation. This is in line with previous studies that have shown no effect on Stroop performance after highfrequency rTMS over the left DLPFC (Vanderhasselt et al., 2006; Wagner et al., 2006).

Although a control task for motor impulsiveness was included in the experiment, no second task to control for DLPFC activation was included, and we thus cannot rule out the possibility that the effects observed might be because of other DLPFC functions (such as working memory, sequencing ability, planning ability, etc.). Additional studies should include such control tasks to further clarify the underlying mechanism for the observed effects and provide additional insights regarding anatomical and functional specificity of the stimulation.

Our results support the investigation of modulation of DLPFC for treatment in pathological high-risk takers, such as individuals with addiction. Patients with addiction show excessively risky decision making (Grant et al., 2000; Bechara et al., 2001; Epstein et al., 2006) that can be captured by the BART (Lejuez et al., 2003a). Functional neuroimaging studies have revealed fairly consistent data indicating abnormal activity in the prefrontal cortex, including the DLPFC, in individuals with nicotine, drug, and food craving (Maas et al., 1998; Goldstein and Volkow, 2002; Wilson et al., 2004). Preliminary studies suggest that neuromodulation of activity in the DLPFC can diminish craving for nicotine (Johann et al., 2003, Eichhammer et al., 2003), cocaine (Camprodon et al., 2007), and food (Uher et al., 2005). Of particular interest, such therapeutic applications require multiple sessions of stimulation of consecutive days. The effects of stimulation on behavioral measures (such as the BART) may provide a predictive marker of desirable therapeutic outcomes. Our results showed that bilateral modulation of DLPFC modified risk-taking behavior significantly. Additional experiments are warranted to explore the effects of cathodal stimulation over the DLPFC coupled with anodal stimulation over other brain areas (e.g., supraorbital region), as well as using different electrode sizes for the reference electrode, such as $100 \mathrm{~cm}^{2}$ (Nitsche et al., 2007), on risk-taking behaviors.

\section{References}

Aklin WM, Lejuez CW, Zvolensky MJ, Kahler CW, Gwadz M (2005) Evaluation of behavioral measures of risk taking propensity with inner city adolescents. Behav Res Ther 43:215-228.

Antal A, Nitsche MA, Kruse W, Kincses TZ, Hoffman KP, Paulus W (2004) Direct current stimulation over V5 enhances visuomotor coordination by improving motion perception in humans. J Cogn Neurosci 16:521-527.

Bechara A, Damasio AR, Damasio H, Anderson SW (1994) Insensitivity to future consequences following damage to human prefrontal cortex. Cognition 50:7-15.

Bechara A, Dolan S, Denburg N, Hindes A, Anderson SW, Nathan PE (2001) Decision-making deficits, linked to a dysfunctional ventromedial prefrontal cortex, revealed in alcohol and stimulant abusers. Neuropsychologia 39:376-389.

Bechara A, Damasio H, Tranel D, Damasio AR (2005) The Iowa gambling task and the somatic marker hypothesis: some questions and answers. Trends Cogn Sci 9:159-162.

Boggio PS, Ferrucci R, Rigonatti SP, Covre P, Nitsche M, Pascual-Leone A, Fregni F (2006) Effects of transcranial direct current stimulation on working memory in patients with Parkinson's disease. J Neurol Sci 249:31-38.

Camprodon JA, Martinez-Raga J, Alonso-Alonso M, Shih MC, PascualLeone A (2007) One session of high frequency repetitive transcranial magnetic stimulation (rTMS) to the right prefrontal cortex transiently reduces cocaine craving. Drug Alcohol Depend 86:91-94.

Clark L, Manes F, Antoun N, Sahakian BJ, Robbins TW (2003) The contributions of lesion laterality and lesion volume to decision-making impairment following frontal lobe damage. Neuropsychologia 41:1474-1483.

Damasio AR, Damasio H, Christen Y (1996) Neurobiology of decisionmaking. Berlin and New York: Springer.

Eichhammer P, Johann M, Kharraz A, Binder H, Pittrow D, Wodarz N, Hajak G (2003) High-frequency repetitive transcranial magnetic stimulation decreases cigarette smoking. J Clin Psychiatry 64:951-953.

Elliot R, Deakin B (2005) Role of the orbitofrontal cortex in reinforcement processing and inhibitory control: evidence from functional magnetic resonance imaging studies in healthy human subjects. Int Rev Neurobiol 65:89-116.

Epstein JA, Bang H, Botvin GJ (2007) Which psychosocial factors moderate or directly affect substance use among inner-city adolescents? Addict Behav 32:700-713.

Ernst M, Paulus MP (2005) Neurobiology of decision making: a selective review from neurocognitive and clinical perspective. Biol Psychiatry 58:597-604. 
Ernst M, Bolla K, Mouratidis M, Contoreggi C, Matochik JA, Kurian V, Cadet JL, Kimes AS, London ED (2002) Decision-making in a risk-taking task. A PET Study. Neuropsychopharmacology 26:682-691.

Fellows LK, Farah MJ (2005) Different underlying impairments in decisionmaking following ventromedial and dorsolateral frontal lobe damage in humans. Cereb Cortex 15:58-63.

Fishbein DH, Eldreth DL, Hyde C, Matochik JA, London ED, Contoreggi C, Kurian V, Kimes AS, Breeden A, Grant S (2005) Risky decision making and the anterior cingulate cortex in abstinent drug abusers and nonusers. Cogn Brain Res 23:119-136.

Fregni F, Boggio PS, Nitsche M, Bermpohl F, Antal A, Feredoes E, Marcolin MA, Rigonatti SP, Silva MTA, Paulus W, Pascual-Leone A (2005) Anodal transcranial direct current stimulation of prefrontal cortex enhances working memory. Exp Brain Res 166:23-30.

Gandiga PC, Hummel FC, Cohen LG (2006) Transcranial DC stimulation (tDCS): a tool for double-blind sham-controlled clinical studies in brain stimulation. Clin Neurophysiol 117:845-850.

Ghashghaei HT, Barbas H (2002) Pathways for emotion: interactions of prefrontal and anterior temporal pathways in the amygdala of the rhesus monkey. Neuroscience 115:1261-1279.

Goldstein RZ, Volkow ND (2002) Drug addiction and its underlying neurobiological basis: neuroimaging evidence for the involvement of the frontal cortex. Am J Psychiatry 159:1642-1652.

Grant S, Contoreggi C, London ED (2000) Drug abusers show impaired performance in a laboratory test of decision making. Neuropsychologia 38:1180-1187.

Iyer MB, Mattu U, Grafman J, Lomarev M, Sato S, Wassermann EM (2005) Safety and cognitive effect of frontal DC brain polarization in healthy individuals. Neurology 64:872-875.

Johann M, Wiegand R, Kharraz A, Bobbe G, Sommer G, Hajak G, Wodarz N, Eichhammer P (2003) [Transcranial magnetic stimulation for nicotine dependence]. Psychiatr Prax 30 [Suppl 2]:S129-S131.

Kincses TZ, Antal A, Nitsche MA, Bartfai O, Paulus W (2004) Facilitation of probabilistic classification learning by transcranial direct current stimulation of the prefrontal cortex in the human. Neuropsychologia 42:113-117.

Knoch D, Gianotti LR, Pascual-Leone A, Treyer V, Regard M, Hohmann M, Brugger P (2006) Disruption of right prefrontal cortex by low-frequency repetitive transcranial magnetic stimulation induces risk-taking behavior. J Neurosci 26:6469-6472.

Krain AL, Wilson AM, Arbuckle R, Castellanos FX, Milham MP (2006) Distinct neural mechanisms of risk and ambiguity: a meta-analysis of decision-making. NeuroImage 32:477-484.

Lejuez CW, Read JP, Kahler CW, Richards JR, Ramsey SE, Stuart GL, Strong DR, Brown R (2002) Evaluation of a behavioral measure of risk-taking: the balloon analogue risk task (BART). J Exp Psychol Appl 6:75-84.

Lejuez CW, Aklin WM, Jones HA, Strong DR, Kahler CW, Read JP (2003a) The balloon analogue risk task (BART) differentiates smokers and nonsmokers. Exp Clin Psychopharmacol 11:26-33.

Lejuez CW, Aklin WM, Zvolensky MJ, Pedulla CM (2003b) Evaluation of the balloon analogue risk task (BART) as a predictor of adolescent realworld risk-taking behaviours. J Adolesc 26:475-479.

Lejuez CW, Simmons BL, Aklin WM, Daughters SB, Dvir S (2004) Risktaking propensity and risky sexual behavior of individuals in residential substance use treatment. Addict Behav 29:1643-1647.

Lejuez CW, Aklin WM, Bornovalova MA, Moolchan E (2005) Differences in risk-taking propensity across inner-city adolescent ever- and neversmokers. Nicotine Tob Res 7:71-79.

Liebetanz D, Nitsche MA, Tergau F, Paulus W (2002) Pharmacological approach to the mechanisms of transcranial DC-stimulation-induced after effects of human motor cortex excitability. Brain 125:2238-2247.

Maas LC, Lukas SE, Kaufman MJ, Weiss RD, Daniels SL, Rogers VW, Kukes TJ, Renshaw PF (1998) Functional magnetic resonance imaging of human brain activation during cue-induced cocaine craving. Am J Psychiatry 155:124-126.

Manes F, Sahakian B, Clark L, Rogers R, Antoun N, Aitken M, Robbins T (2002) Decision-making processes following damage to the prefrontal cortex. Brain 125:624-639.

Marshall L, Mölle M, Siebner HR, Born J (2005) Bifrontal transcranial direct current stimulation slows reaction time in a working memory task. BMC Neuroscience 6:23

Navqi N, Tranel D, Bechara A (2006) Visceral and decision-making func- tions of the ventromedial prefrontal cortex. In: The orbitofrontal cortex (Zald DH, Rauch SL, eds), pp 325-353. Oxford: Oxford UP.

Nitsche MA, Paulus W (2000) Excitability changes induced in the human motor cortex by weak transcranial direct current stimulation. J Physiol (Lond) 527:633-639.

Nitsche MA, Paulus W (2001) Sustained excitability elevations induced by transcranial DC motor cortex stimulation in humans. Neurology 57:1899-1901.

Nitsche MA, Liebetanz D, Antal A, Lang N, Tergau F, Paulus W (2003a) Modulation of cortical excitability by weak direct current stimulation technical, safety and functional aspects. Suppl Clin Neurophysiol 56:255-276.

Nitsche MA, Schauenburg A, Lang N, Liebetanz D, Exner C, Paulus W, Tergau F (2003b) Facilitation of implicit motor learning by weak transcranial direct current stimulation of the primary motor cortex in the human. J Cog Neurosci 15:619-626.

Nitsche MA, Doemkes S, Karakoese T, Antal A, Liebetanz D, Lang N, Tergau F, Paulus W (2007) Shaping the effects of transcranial direct current stimulation of the human motor cortex. J Neurophysiol 97:3109-3117.

Paulus MP, Hozack N, Zauscher B, McDowell JE, Frank L, Brown GG, Braff DL (2001) Prefrontal, parietal, and temporal cortex networks underlie decision-making in the presence of uncertainty. NeuroImage 13:91-100.

Priori A (2003) Brain polarization in humans: a reappraisal of an old tool for prolonged non-invasive modulation of brain excitability. Clin Neurophysiol 114:589-595.

Purpura D, McMurtry J (1965) Intracellular activities and evoked potential changes during polarization of motor cortex. J Neurophysiol 28:166-185

Rafal R (2001) Virtual neurology. Nat Neurosci 4:862-864.

Robertson EM, Théoret H, Pascual-Leone A (2003) Studies in cognition: the problems solved and created by transcranial magnetic stimulation. J Cogn Neurosci 15:948-960.

Rogers RD, Owen AM, Middleton HC, Williams EJ, Pickard JD, Sahakian BJ, Robins TW (1999) Choosing between small, likely rewards and large, unlikely rewards activates inferior and orbital prefrontal cortex. J Neurosci 20:9029-9038.

Romero JR, Anschel D, Sparing R, Gangitano M, Pascual-Leone (2002) Subthreshold low frequency repetitive transcranial magnetic stimulation selectively decreases facilitation in the motor cortex. Clin Neurophysiol 113(1):101-107.

Sanfey AG, Rilling KJ, Aronson JA, Nystrom LE, Cohen JD (2003) The neural basis of economic decision-making in the Ultimate Game. Science 300:1673-1675.

Smith K, Dickhaut J, McCabe K, Pardo J (2002) Neuronal substrates for choice under ambiguity, risk, certainty, gains, and losses. Manage Sci 48:711-718.

Stroop JR (1935) Studies of interference in serial verbal reactions. J Exp Psychol 18:643-662.

Tranel D, Bechara A, Denburg NL (2002) Asymmetric functional roles of right and left ventromedial prefrontal cortices in social conduct, decisionmaking, and emotional processing. Cortex 38:589-612.

Tranel D, Damasio H, Denburg NL, Bechara A (2005) Does gender play a role in functional asymmetry of ventromedial prefrontal cortex? Brain 128:2872-2881.

Uher R, Yoganathan D, Mogg A, Eranti SV, Treasure J, Campbell IC, McLoughlin DM, Schmidt U (2005) Effect of left prefrontal repetitive transcranial magnetic stimulation on food craving. Biol Psychiatry $58: 840-842$.

Vanderhasselt MA, De Raedt R, Baeken C, Leyman L, D’Haenen H (2006) The influence of rTMS over the left dorsolateral prefrontal cortex on Stroop task performance. Exp Brain Res 169:279-282.

van't Wout M, Kahn RS, Sanfey AG, Aleman A (2005) Repetitive transcranial magnetic stimulation over the right dorsolateral prefrontal cortex affects strategic decision-making. NeuroReport 16:1849-1852.

Wagner M, Rihs TA, Mosimann UP, Fisch UA, Schlaepfer TE (2006) Repetitive transcranial magnetic stimulation of the dorsolateral prefrontal cortex affects divided attention immediately after cessation of stimulation. J Psychiatr Res 40:315-321.

Wassermann EM, Grafman J (2005) Recharging cognition with DC brain polarization. Trends Cogn Sci 9:503-505.

Wilson SJ, Sayette MA, Fiez JA (2004) Prefrontal responses to drug cues: a neurocognitive analysis. Nat Neurosci 7:211-214.

Zak PJ (2004) Neuroeconomics. Philos Trans R Soc Lond B Biol Sci 359: $1737-1748$. 